\title{
Closed Form Isoparametric Shape Functions of Four-node Convex Finite Elements
}

\author{
Gautam Dasgupta, Member ASCE \\ Columbia University, New York, NY 10027, USA \\ dasgupta@columbia.edu
}

\section{Key words:}

Closed form shape functions, exact integration, four node triangles, high accuracy finite elements, isoparametric forms, Taig shape functions, Wachspress interpolants.

\begin{abstract}
On arbitrary plane quadrilaterals, difficulties in integrating energy densities prevented analysts from directly using shape function expressions in terms of the physical coordinate variables $(x$ and $y)$. With the availability of an exact integration procedure, shape functions are sought here as explicit expressions (in $x$ and $y$ ). Conventional isoparametric (indirect) representation, via canonical coordinate variables $(\eta$ and $\xi$ ) on a unit square in the computational domain, do not reveal the presence of irrational algebraic expressions that were first elaborated by Wachspress. Computer algebra systems demonstrate that the isoparametric shape functions are: for a general quadrilateral linear and a square root of a quadratic (in $x$ and $y$ ); for a trapezoid - identical to the Wachspress rational polynomials; for a parallelogram - bilinear functions; and, moreover, even valid for a triangle
\end{abstract}


with a side node. The failure of the isoparametric formulation for concave domains is traced to the negative argument of irrational parts. Shape functions in the physical domain $(x-$ and $y-)$ facilitate contour plotting of responses (e.g., temperature distributions) within quadrilateral elements. A subsequent paper details exact calculation of stiffness matrices where the presented shape functions (in $x$ and $y$ ) are indispensable.

May 24, 2006 


\section{Introduction}

Generation of shape functions is the most fundamental task in any finite element implementation. Following Courant's formulation, ref. [2], for twoand three-dimensional simplex elements, i.e., triangles and tetrahedrons respectively, the shape functions are linear algebraic functions of coordinate variables, $x, y$ and $x, y, z$ respectively, of the form:

$$
\begin{aligned}
& a_{o}+a_{1} x+a_{2} y=0 ; \quad \text { for two-dimensional cases } \\
& a_{o}+a_{1} x+a_{2} y+a_{3} z=0 ; \quad \text { for three-dimensional cases }
\end{aligned}
$$

The challenge to postulate shape functions for plane quadrilaterals and threedimensional hexahedrals were adequately met by the ingenious formulation of Taig, ref. [9] — well-known in the literature as the isoparametric formulation. This algebraic construct is the focus of this paper. In essence, Taig starts with the bi-linear or tri-linear forms of shape functions on a unit square or a unit cube for two- or three-dimensions, respectively, in $(\eta, \xi)$ and $(\eta, \xi, \zeta)$ Cartesian coordinates. The isoparametric assumption is that the physical coordinates $(x, y)$ and $(x, y, z)$, which define the actual element in space, are themselves 'interpolated' from the nodal values of elements. Details can be found in standard text books, e.g., ref. [13]. However, in no literature shape functions are expressed in $(x, y)$ and $(x, y, z)$ coordinates. The objective of this paper is to furnish explicit algebraic expressions for isoparametric shape functions in terms of $(x, y)$ coordinates for plane convex quadrilaterals. Similar methodology is anticipated to yield shape functions for analogous three-dimensional cases. 


\subsection{Essential properties of shape functions}

Shape functions are approximating test functions for elliptic partial differential equations of mathematical physics, e.g., potential and vibration problems, ref. [2]. These approximants are in accordance with the nodal independence characterization. For a generic node (number $i$ ), whose coordinates are represented by a location vector $p_{i}-\left(x_{i}, y_{i}\right),\left(x_{i}, y_{i}, z_{i}\right)$ for two- and three dimensional cases, respectively — a shape function $\phi_{i}$ possesses the following global Kronecker property:

$$
\begin{aligned}
\phi_{i}\left(p_{j}\right) & =\delta_{i j}: \text { Kronecker's delta, i.e., } \\
& =1, \text { for } i=j \\
& =0, \text { for } i \neq j
\end{aligned}
$$

The elliptic nature of the field equation necessitates strict adherence to the following local maximum-minimum condition and the mean-value form:

$$
0<\phi(p)<1, p \in \Omega, \quad \text { and } \sum \phi_{j}(p)=1
$$

respectively, within the element domain $\Omega$.

An objectivity condition stems from the second order partial differential equations that mandates exact interpolation of a constant and linear fields:

$$
\begin{aligned}
\sum x_{j} \phi_{j}(p) & =x ; \sum y_{j} \phi_{j}(p)=y \\
\text { and } \sum z_{j} \phi_{j}(p) & =z \text { - for three-dimensional problems }
\end{aligned}
$$


A linear field between nodes is sufficient to prevent inter-element separation (i.e., a finite jump in the field value across element interfaces):

$$
\begin{aligned}
& \phi_{i}(p)=1-\tau ; \\
& \tau \text { measured from } p_{i}(\tau=0) \text { to } p_{i \pm 1}(\tau=1)
\end{aligned}
$$

The above prescription for shape functions are for second order partial differential equations, higher (even) order cases can be similarly constructed for more complex elements with adequate number of degrees-of-freedom.

A replica of equation (5) with $\Phi$ - the shape functions on the canonical square or cube where a generic point $p^{*}$ denotes $(\eta, \xi)$ or $(\eta, \xi \zeta)$, respectively, becomes:

$$
\begin{array}{r}
\sum x_{j} \Phi_{j}\left(p^{*}\right)=x ; \sum y_{j} \Phi_{j}\left(p^{*}\right)=y \\
\text { and } \sum z_{j} \Phi_{j}\left(p^{*}\right)=z-\text { for three-dimensions }
\end{array}
$$

This is the cardinal idea behind the isoparametric formulation. This ingenious algebraic scheme (even though devoid of any geometrical basis) has elevated the finite element method beyond triangulation.

\subsection{The Wachspress formulation}

Due to the lack of any analytical reasoning behind the isoparametric formulation, such a useful method cannot be extended to convex polygons of arbitrary number of sides. Wachspress, ref. [12], employed projective geometry constructs to generate shape functions for all convex polygons in the form of rational polynomials, i.e., a polynomial divided by another polynomial (of 
one less degree). A computational treatment can be found in ref. [4]. For four-node quadrilaterals a shape function is of the following form:

$$
\frac{a_{o}+a_{1} x+a_{2} y+a_{3} x^{2}+a_{4} x y+a_{5} y^{2}}{b_{o}+b_{1} x+b_{2} y} ; a_{i}, b_{j}: \text { constants }
$$

It is demonstrated in this paper that the isoparametric formulation, though heuristic, yet reproduces the Wachspress results for trapezoids (and parallelograms). An interesting fact, which is illustrated here, is that for a triangle with a side node, equation (8) is not valid. However, the isoparametric formulation still holds. These new results, which are reported here for the first time, became available when the isoparametric idea has been explored in the $(x, y)$ (physical) coordinates rather using the conventional $(\eta, \xi)$ canonical variables.

A computer algebra environment, e.g., Mathematica, becomes indispensable to constructing shape function in the (object) physical $(x, y)$ coordinates. Detailed steps for hand calculation (and verification) are presented here along with suggested strategies to conveniently develop efficient $\mathrm{C}$ or FORTRAN programs (from the Mathematica symbolic code segments). 


\section{Closed form expressions for isoparametric coordinate transformations}

The isoparametric formulation yields shape functions for irregular convex quadrilaterals (hereafter referred as quadrilaterals and convex unless otherwise explicitly indicated). The starting point is the bilinear shape functions on a unit square (the computational domain) in Figure 1 with corner nodes at:

$$
(0,0), \quad(1,0), \quad(1,1), \quad(0,1)
$$

in $(\eta, \xi)$ coordinates.
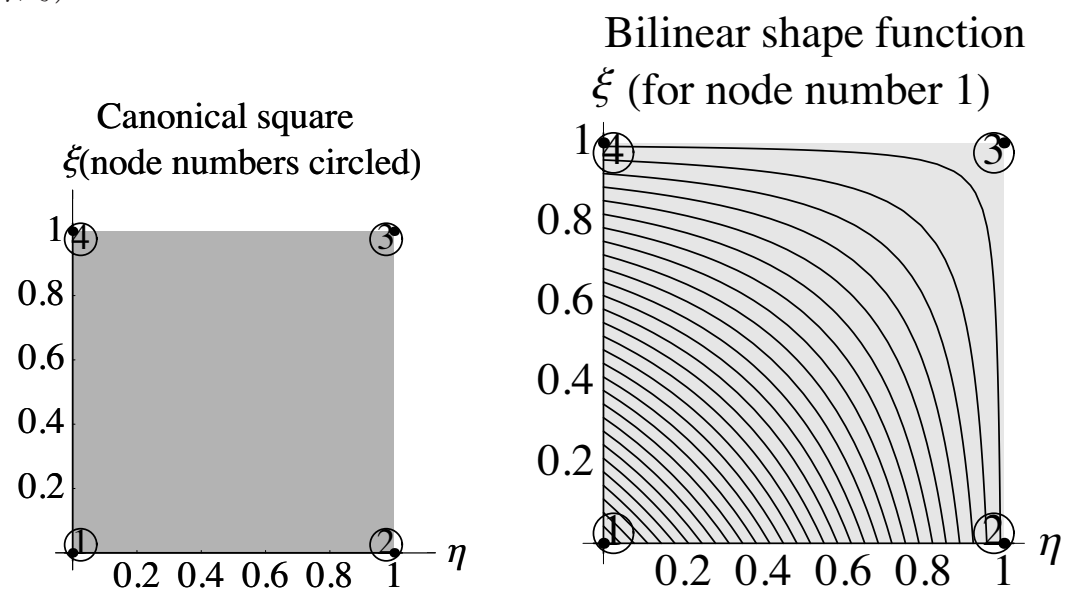

Figure 1: Canonical square - computational domain

In terms of the canonical $(\eta, \xi)$ coordinate variables the shape functions are:

$$
(1-\eta)(1-\xi), \quad \eta(1-\xi), \quad \eta \xi, \quad(1-\eta) \xi
$$

The contour plot of the first one is shown in Figure 1. 
Consider an arbitrary (convex) quadrilateral in the $x-y$ plane with the following vertices (numbered counterclockwise), vide Figure 2:

$$
\left(x_{1}, y_{1}\right) ; \quad\left(x_{2}, y_{2}\right) ; \quad\left(x_{3}, y_{3}\right) ; \quad\left(x_{4}, y_{4}\right)
$$

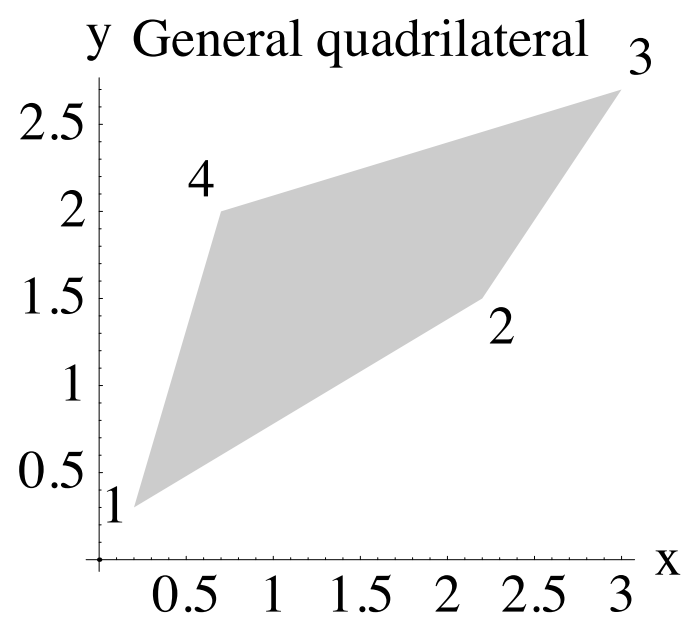

Figure 2: A general quadrilateral

The isoparametric formulation estimates the $x$ and $y$ coordinates in terms of the expressions in (10) and (11). These coordinates (both $x$ and $y$ ) themselves are conceived as variables to be interpolated from the nodal values stated in (11) leading to:

$$
\begin{aligned}
& x=(1-\eta)(1-\xi) x_{1}+\eta(1-\xi) x_{2}+\eta \xi x_{3}+(1-\eta) \xi x_{4} \\
& y=(1-\eta)(1-\xi) y_{1}+\eta(1-\xi) y_{2}+\eta \xi y_{3}+(1-\eta) \xi y_{4}
\end{aligned}
$$

From the above two bilinear equations one can eliminate $\eta$ and obtain the 
following quadratic equation in $\xi$ :

$$
\begin{aligned}
\alpha_{\xi} \xi^{2} & +\beta_{\xi} \xi+\gamma_{\xi}=0, \text { where the coefficients are: } \\
\alpha_{\xi}=- & x_{2} y_{1}+x_{3} y_{1}+x_{1} y_{2}-x_{4} y_{2}-x_{1} y_{3} \\
& +x_{4} y_{3}+x_{2} y_{4}-x_{3} y_{4} \\
\beta_{\xi}= & y x_{1}-y x_{2}+y x_{3}-y x_{4}-x y_{1}+2 x_{2} y_{1} \\
& -x_{3} y_{1}+x y_{2}-2 x_{1} y_{2}+x_{4} y_{2}-x y_{3} \\
& +x_{1} y_{3}+x y_{4}-x_{2} y_{4} \\
\gamma_{\xi}= & -y x_{1}+y x_{2}+x y_{1}-x_{2} y_{1}-x y_{2}+x_{1} y_{2}
\end{aligned}
$$

To facilitate hand calculation and verification the aforementioned relations are now illustrated for a particular quadrilateral, shown in Figure 2, whose vertices are:

$$
\left(\frac{1}{5}, \frac{3}{10}\right) ;\left(\frac{11}{5}, \frac{3}{2}\right) ; \quad\left(3, \frac{27}{10}\right) ; \quad\left(\frac{7}{10}, 2\right)
$$

The interpolated $x$ and $y$ coordinates in terms of the canonical shape functions, as depicted in equations (12) and (13), are:

$$
\begin{aligned}
& x=\frac{(1-\eta)(1-\xi)}{5}+\frac{11 \eta(1-\xi)}{5}+\frac{7(1-\eta) \xi}{10}+3 \eta \xi \\
& y=\frac{3(1-\eta)(1-\xi)}{10}+\frac{3 \eta(1-\xi)}{2}+2(1-\eta) \xi+\frac{27 \eta \xi}{10}
\end{aligned}
$$

Elimination of $\eta$ from the above two equations leads to:

$$
76 \xi^{2}+(299-50 x-30 y) \xi+(36+120 x-200 y)=0
$$

It is important to note that the roots of the above quadratic equation are real within the element domain. The discriminant of equation (19) vanishes 
along the 'zero discriminant curve' as shown in Figure 3. Consider two sample points situated in two different segments that are separated by the curve. The values of the discriminant:

$(299-50 x-30 y)^{2}-76 \times 4(36+120 x-200 y)$

on the upper left side of the curve, at $(1,0)$ is $14577>0$ and on the lower right at $(3,0)$ is $-998183<0$, are of different signs.

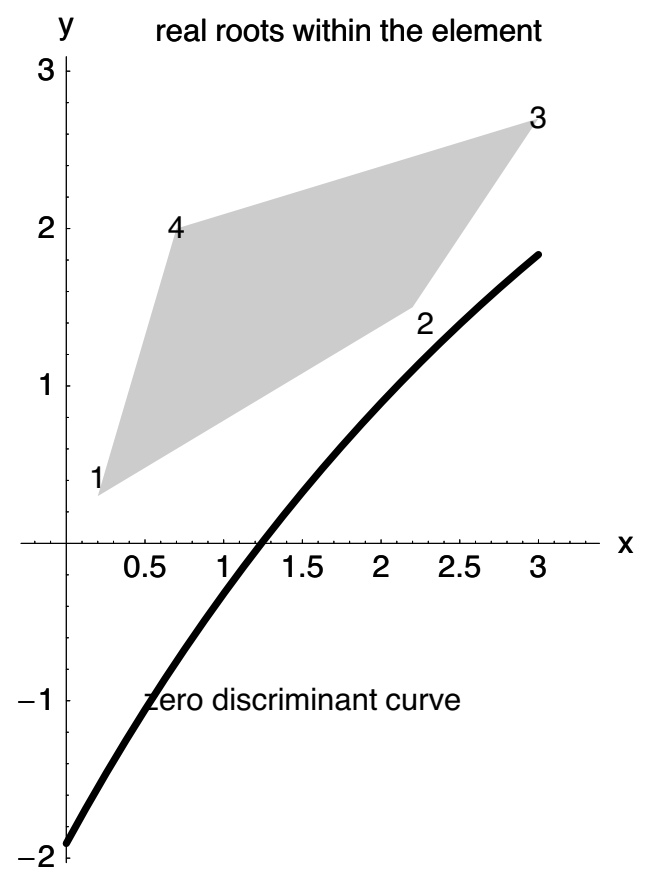

Figure 3: Zero discriminant in the physical coordinates 


\subsection{Robustness of the isoparametric formulation}

The nature of the solutions for $\eta$ and $\xi$ govern the shape function expressions in the $x$ and $y$ coordinates. Thus, one constructs the following quadratic equation, analogous to equation (14), in terms of $\eta$. Eliminating $\xi$ from equations (17) and (18) one obtains:

$$
\begin{aligned}
\alpha_{\eta} \eta^{2}+\beta_{\eta} \eta & +\gamma_{\eta}=0 \\
\text { where } \alpha_{\eta}= & x_{3} y_{1}-x_{4} y_{1}-x_{3} y_{2}+x_{4} y_{2}-x_{1} y_{3} \\
& +x_{2} y_{3}+x_{1} y_{4}-x_{2} y_{4} \\
\beta_{\eta}= & y x_{1}-y x_{2}+y x_{3}-y x_{4}-x y_{1}-x_{3} y_{1} \\
& +2 x_{4} y_{1}+x y_{2}-x_{4} y_{2}-x y_{3}+x_{1} y_{3} \\
& +x y_{4}-2 x_{1} y_{4}+x_{2} y_{4} \\
\gamma_{\eta}= & -y x_{1}+y x_{4}+x y_{1}-x_{4} y_{1}-x y_{4}+x_{1} y_{4}
\end{aligned}
$$

The discriminants calculated from equations (20) and (21) are identical to the one shown in Figure 3, since $\beta^{2}-4 \alpha \gamma$ is the invariant for the coordinate transformation. For the particular element, with the nodal coordinates denoted in equation (16), the quadratic equation in $\eta$ becomes:

$$
136 \eta^{2}-(261+50 x+30 y) \eta+(-19+170 x-50 y)=0
$$

The discriminant obtained from the above equation is of the form $\mathcal{A} x^{2}+$ $\mathcal{B} x y+\mathcal{C} y^{2}+$ linear terms, which in explicit terms becomes:

$$
2500 x^{2}+3000 x y+900 y^{2}-66380 x+42860 y+78457
$$


This represents a parabolic curve in $x-y$ plane since:

$\mathcal{B}^{2}-4 \mathcal{A C}=(3000)^{2}-4 \times 2500 \times 900=0$.

In general, from equations (14) and (20), the discriminant can be structured in the following form:

$$
\begin{aligned}
\beta_{\eta}^{2}-4 \alpha_{\eta} \gamma_{\eta} & =\beta_{\xi}^{2}-4 \alpha_{\xi} \gamma_{\xi} \text { i.e., } \beta^{2}-4 \alpha \gamma \\
& =\mathcal{A} x^{2}+\mathcal{B} x y+\mathcal{C} y^{2}+\text { lower order terms }
\end{aligned}
$$

where $\mathcal{A}=\left(y_{1}-y_{2}+y_{3}-y_{4}\right)^{2}$

$$
\begin{aligned}
& \mathcal{B}=-2\left(x_{1}-x_{2}+x_{3}-x_{4}\right)\left(y_{1}-y_{2}+y_{3}-y_{4}\right) \\
& \mathcal{C}=\left(x_{1}-x_{2}+x_{3}-x_{4}\right)^{2}
\end{aligned}
$$

since $\left(\mathcal{B}^{2}-4 \mathcal{A C}\right)$ is always zero the $x-$ and $y-$ plot for $\left(\beta^{2}-4 \alpha \gamma\right)$ is a parabola (for non-trivial cases). Hence in Figure 3 the zero discriminant curve can never enter into the element domain. This asserts the robustness of the isoparametric formulation for (convex) quadrilaterals.

\section{Coefficients of quadratic equations govern- ing the canonical parameters $\eta$ and $\xi$}

The isoparametric shape functions in equation (10) are expressed in $\eta$ and $\xi$ as a full bi-linear set. The parameters $\eta-$ and $\xi$ - can be solved as the roots of equations (14) and (20) in terms of the location $(x, y)$ and the nodal coordinates with numeric values for variables in expression (11). Thus the shape functions for a convex quadrilateral can be obtained in terms of the nodal coordinates as algebraic expressions of $(x, y)$, as illustrated in the aforementioned example. 
It is possible to facilitate solutions of $(\eta, \xi)$ by expressing the coefficients of quadratics in equations (14) and (20). All such coefficients can be explicitly obtained in terms of triangular areas:

$$
\begin{aligned}
\Delta_{i j k} & =\operatorname{det}\left[\begin{array}{c}
1,1,1 \\
x_{i}, x_{j}, x_{k} \\
y_{i}, y_{j}, y_{k}
\end{array}\right] \\
& =\text { twice the area of the triangle with vertices: }\left(x_{i}, y_{i}\right),\left(x_{j}, y_{j}\right),\left(x_{k}, y_{k}\right) \\
& =\left(-x_{j} y_{i}+x_{k} y_{i}+x_{i} y_{j}-x_{k} y_{j}-x_{i} y_{k}+x_{j} y_{k}\right) \\
& i, j, k: 1,2,3,4 \text { for nodes, and } \\
& : 0 \text { denotes a generic point }(x, y) \text { within the element }
\end{aligned}
$$

By combining five indices: $i, j, k=0,1,2,3,4$ and taking a group of three to calculate equation $(27)$ there are $\left({ }^{5} C_{3}=\right) 10$ terms whose linear combinations express: $\left(\alpha_{\eta}, \beta_{\eta}, \gamma_{\eta}\right)$ and $\left(\alpha_{\xi}, \beta_{\xi}, \gamma_{\xi}\right)$ of equations (15) and (21) as elaborated below. 


\subsection{Coefficients of quadratic equations for $\mathrm{C}, \mathrm{C}^{++}$and FORTRAN coding}

Here the coefficients of the quadratic equations, equations (14) and (20), are presented in the expanded form:

$$
\begin{aligned}
\alpha_{\xi} & =-x_{2} y_{1}+x_{3} y_{1}+x_{1} y_{2}-x_{4} y_{2}-x_{1} y_{3}+x_{4} y_{3}+x_{2} y_{4}-x_{3} y_{4} \\
& =\Delta_{4,1,2}-\Delta_{3,4,1} \\
\gamma_{\xi} & =-y x_{1}+y x_{4}+x y_{1}-x_{4} y_{1}-x y_{4}+x_{1} y_{4} \\
& =\Delta_{0,1,2} \\
\alpha_{\eta} & =x_{3} y_{1}-x_{4} y_{1}-x_{3} y_{2}+x_{4} y_{2}-x_{1} y_{3}+x_{2} y_{3}+x_{1} y_{4}-x_{2} y_{4} \\
& =\Delta_{2,3,4}-\Delta_{3,4,1} \\
\gamma_{\eta} & =\Delta_{4,0,1}
\end{aligned}
$$

The (middle) $\beta$ terms are somewhat involved but can be verified from equations (15) and (21) to be:

$$
\beta_{\xi}=-\Delta_{1,2,0}-\Delta_{0,3,4}-\Delta_{1,2,3}+\Delta_{4,2,3}
$$

and

$$
\beta_{\eta}=-\Delta_{0,1,2}-\Delta_{0,3,4}+\Delta_{1,2,4}+\Delta_{4,1,3}
$$




\subsection{Degenerated cases of trapezoids}

It is interesting to explore the degenerate quadratics when the leading coefficients, viz., $\alpha_{\eta}, \alpha_{\xi}$, vanish. In such cases there will be no quadratic equation and the canonical parameters $\eta-$ and $\xi-$ will take the form: $-\frac{\gamma}{\beta}$, vide equations (31), (31), (32), (33) and (34). The geometrical implications are traced below.

Let us first consider the case when $\alpha_{\xi}$ in equation (30) vanishes, i.e., when the areas of triangles with vertices 4,1 , and 2 and with vertices 3,4 , and 1 become the same. From Figure 4 one concludes that sides $4-1$ and 2-3 are parallel.
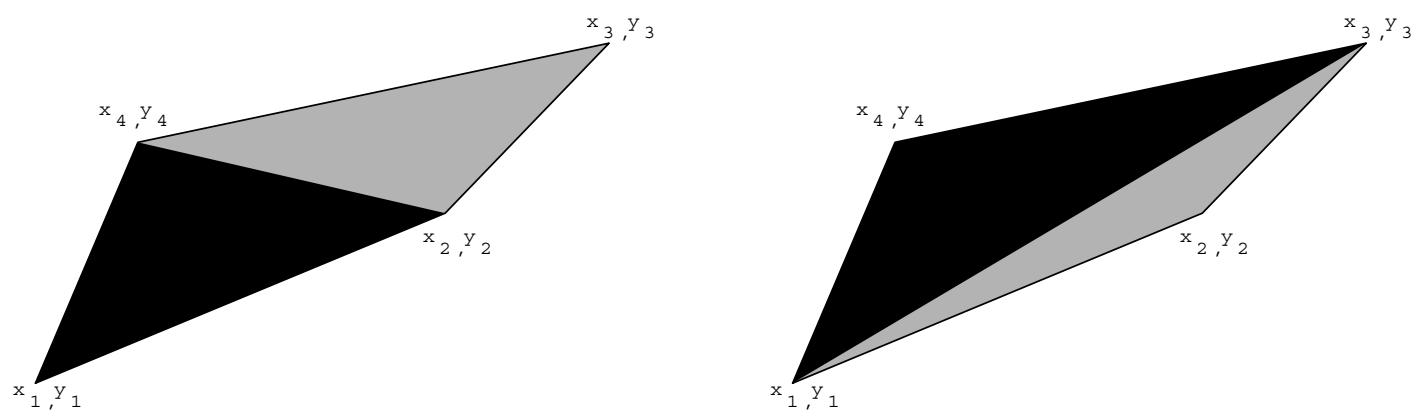

Figure 4: A special quadrilateral: $\alpha_{\xi}=0$

Similarly, $\alpha_{\eta}=0$ implies, vide Figure 5, the sides 1-2 and 3-4 are parallel.
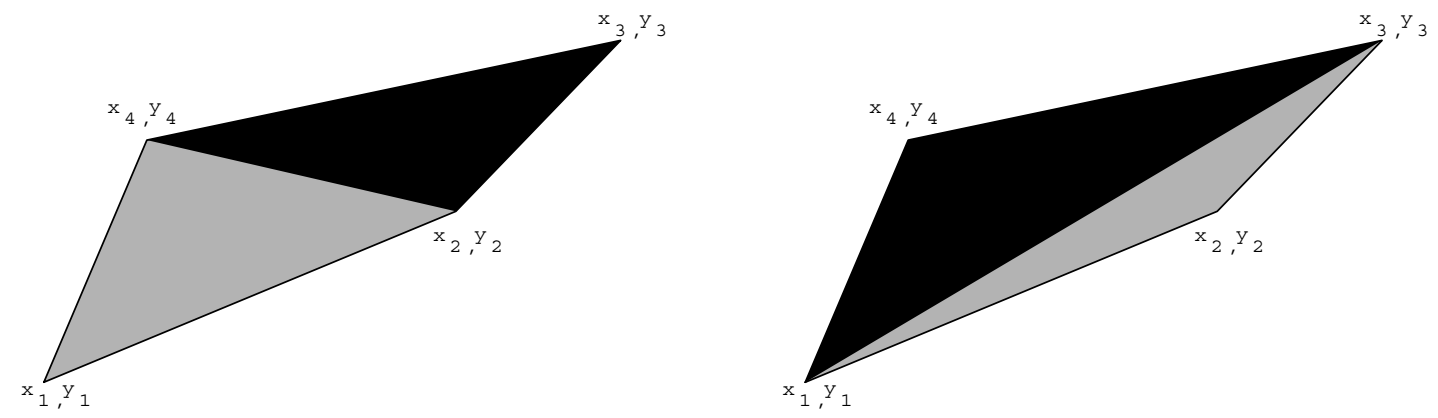

Figure 5: A special quadrilateral: $\alpha_{\eta}=0$ 
These cases of trapezoidal elements are of special significance since experiences with isoparametric quadrilaterals demonstrated a higher degree of accuracy than a quadrilateral of considerable skewness when the popular numerical quadrature is employed in element formulation.

To facilitate finite element code development in procedural (including ob-

ject oriented $\mathrm{C}^{++}$, Java, $\mathrm{C}^{\#}$ ) languages, a benchmark example is illustrated follows.

\section{A special case: trapezoid with two sides parallel to the $x$-axis}

In the literature, Taig's ingenious isoparametric formulation is hardly related to any geometrical concept since all calculations are carried out in the computational square (in $\eta-$ and $\xi-$ ) rather than in the element itself (in $x-$ and $y-$ system). Some new results of this section facilitates bridging the gap between the isoparametric scheme and those based on rigorous analytical concepts, e.g., Wachspress' projective geometry and perspective transformations.

Consider a particular trapezoid:

$$
y_{2}=y_{1} \text { and } y_{4}=y_{3}
$$




\section{A Trapezoid}

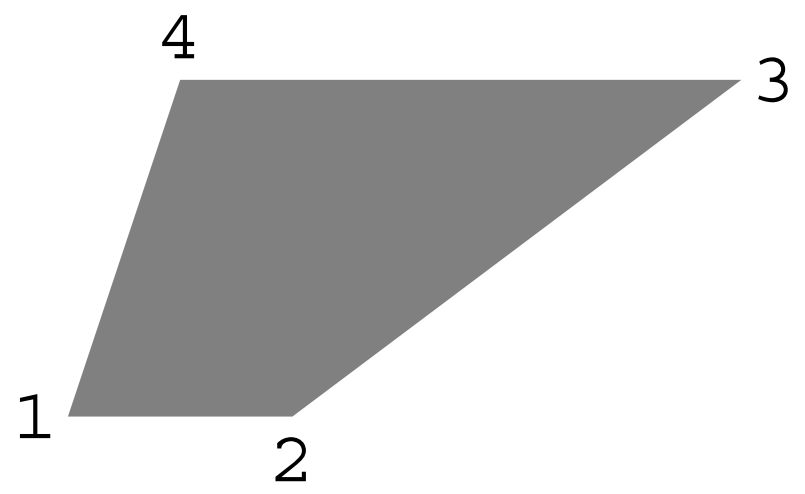

Figure 6: A trapezoid

In this case:

$$
\begin{aligned}
\eta & =\frac{y x_{1}-y x_{4}+x_{4} y_{1}-x_{1} y_{3}+x\left(-y_{1}+y_{3}\right)}{y x_{1}-y x_{2}+y x_{3}-y x_{4}-x_{3} y_{1}+x_{4} y_{1}-x_{1} y_{3}+x_{2} y_{3}} \\
\xi & =\frac{y-y_{1}}{y_{3}-y_{1}}
\end{aligned}
$$

There is no irrational (square root like) term of $\eta$ and $\xi$ in the aforementioned expression.

Observe that $\eta$ is in a rational polynomial (Padé) form. The other coordinate $\xi$ is a linear function in $y$. Thus the terms in equation (10), i.e., $(1-\eta)(1-\xi), \quad \eta(1-\xi), \quad \eta \xi, \quad(1-\eta) \xi$, are all rational polynomials, where the numerator polynomial is second order in $x$ and $y$, and the denominator is a linear function. These shape functions coincide with the Wachspress interpolants, ref. [4] based on the considerations of projective geometry, ref. [11]. This serendipitous identity with the Wachspress values 
explains why trapezoidal elements, in all cases, perform better than general quadrilaterals (under isoparametric modeling).

\subsection{Parallelograms}

The isoparametric mapping becomes identical with the affine transformation, vide ref. [8], for arbitrary parallelograms. This strong geometrical criterion yields excellent accuracy, as has been widely observed, vide ref. [1].

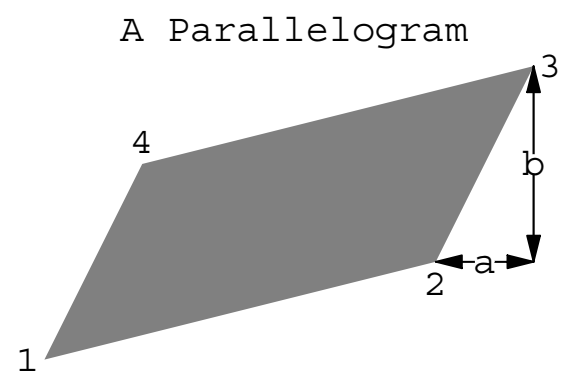

Figure 7: A parallelogram (with variables $a$ and $b$ shown)

The independent variables are the coordinates of the first and second nodes, and the horizontal and vertical offsets $a$ and $b$, respectively, shown in Figure 7 .

The isoparametric equations equations (12) and (13) become:

$$
\begin{aligned}
& x=(1-\eta)(1-\xi) x_{1}+(1-\eta) \xi\left(a+x_{1}\right)+\eta(1-\xi) x_{2}+\eta \xi\left(a+x_{2}\right) \\
& y=(1-\eta)(1-\xi) y_{1}+(1-\eta) \xi\left(b+y_{1}\right)+\eta(1-\xi) y_{2}+\eta \xi\left(b+y_{2}\right)
\end{aligned}
$$

Elimination of $\eta$ leads to:

$$
\begin{aligned}
& -y x_{1}+b \xi x_{1}+y_{2} x_{1}+y x_{2}-b \xi x_{2}-a \xi y_{1}-x_{2} y_{1}+a \xi y_{2} \\
& =x\left(y_{2}-y_{1}\right)
\end{aligned}
$$


Thus:

$$
\xi=-\frac{\gamma}{\beta}=-\frac{-y x_{1}+y_{2} x_{1}+y x_{2}-x_{2} y_{1}-x\left(y_{2}-y_{1}\right)}{b x_{1}-b x_{2}-a y_{1}+a y_{2}}
$$

Similarly after the elimination of $\xi$ the other canonical parameter $\eta$ becomes:

$$
\eta=-\frac{-b x+a y+b x_{1}-a y_{1}}{-b x_{1}+b x_{2}+a y_{1}-a y_{2}}
$$

Now, for an arbitrary (convex) quadrilateral, the shape functions introduced

in equation (3), can be obtained using the bilinear forms in equation (10) as follows:

$$
\begin{aligned}
& \phi_{1}^{*}=\left(b\left(x-x_{2}\right)+a\left(y_{2}-y\right)\right) \phi_{o}^{*} \\
& \phi_{o}^{*}=\left(b\left(x_{1}-x_{2}\right)+x_{2} y-a y_{1}+x y_{1}-x_{2} y_{1}+a y_{2}-x y_{2}+x_{1}\left(y_{2}-y\right)\right) \\
& \phi_{2}^{*}=-\left(b\left(x-x_{1}\right)+a\left(y_{1}-y\right) \phi_{o}^{*}\right. \\
& \left.\phi_{3}^{*}=\left(b\left(x-x_{1}\right)+a\left(y_{1}-y\right)\right)\left(x_{2}\left(y-y_{1}\right)+x\left(y_{1}-y_{2}\right)+x_{1}\left(y_{2}-y\right)\right) y_{2}+x_{1}\left(y_{2}-y\right)\right) \\
& \phi_{4}^{*}=\left(b\left(x-x_{2}\right)+a\left(y_{2}-y\right)\right)\left(x_{2}\left(y_{1}-y\right)+x_{1}\left(y-y_{2}\right)+x\left(y_{2}-y_{1}\right)\right)
\end{aligned}
$$

where

$$
\phi_{1}=\frac{\phi_{1}^{*}}{\mathcal{D}} ; \phi_{2}=\frac{\phi_{2}^{*}}{\mathcal{D}} ; \phi_{3}=\frac{\phi_{3}^{*}}{\mathcal{D}} ; \phi_{4}=\frac{\phi_{4}^{*}}{\mathcal{D}} ;
$$

and the denominator

$$
\mathcal{D}=\left(b\left(x_{1}-x_{2}\right)+a\left(y_{2}-y_{1}\right)\right)^{2}
$$

\section{Interdependency between quadrilateral shape functions}

Symbolic computational environments can take advantage of the fact that only one shape function (out of the four shape functions) is independent. The 
remaining three can be derived from the desirable conditions to reproduce exactly any arbitrary linear field. For the four shape functions, which pertain to a given quadrilateral element, $\phi_{i}, i=1,2,3,4$ the three linear relations from equation (5) are:

$$
\begin{array}{r}
\phi_{1}+\phi_{2}+\phi_{3}+\phi_{4}=1 \\
x_{1} \phi_{1}+x_{2} \phi_{2}+x_{3} \phi_{3}+x_{4} \phi_{4}=x \\
y_{1} \phi_{1}+y_{2} \phi_{2}+y_{3} \phi_{3}+y_{4} \phi_{4}=y
\end{array}
$$

The solution for $\phi_{2}, \phi_{3}, \phi_{4}$ can be obtained in terms of $\phi_{1}$. This computation is not complete since the non-negativity of $\phi_{1}$ within the element (region $\Omega$ ) cannot guarantee the same for $\phi_{2}, \phi_{3}, \phi_{4}$.

The interdependency relation reveals the limitation of non applicability of (non-singular) regular functions to a degenerate case - a triangle with a side node.

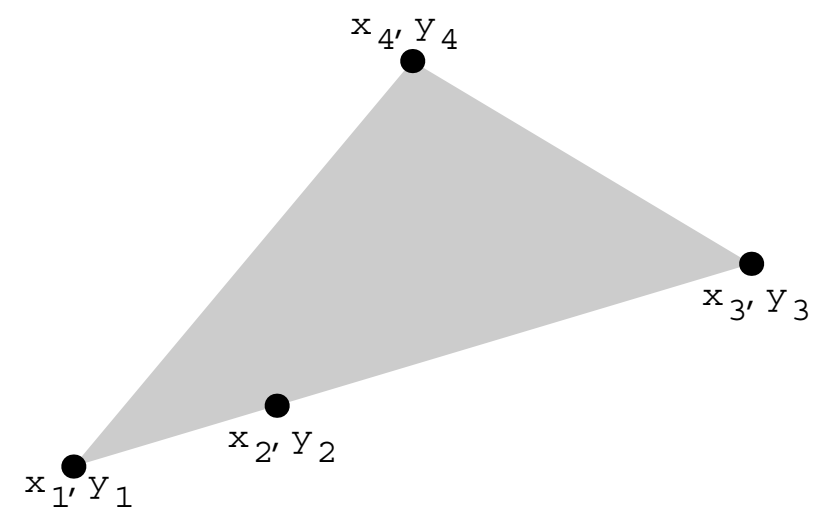

Figure 8: A triangle with a side node - a degenerated quadrilateral 
A matrix form of the linearity, which is expressed by equations (47), (48) and (49), is:

$$
\left[\begin{array}{ccc}
1 & 1 & 1 \\
x_{2} & x_{3} & x_{4} \\
y_{2} & y_{3} & y_{4}
\end{array}\right]\left\{\begin{array}{c}
\phi_{2} \\
\phi_{3} \\
\phi_{4}
\end{array}\right\}=\left\{\begin{array}{c}
1-\phi_{1} \\
x-x_{1} \phi_{1} \\
y-y_{1} \phi_{1}
\end{array}\right\}
$$

When the nodes 2, 3 and 4 are on the same line, then the determinant of the left hand side matrix in equation (50) vanishes. Consequently, any determination of $\phi_{2}, \phi_{3}, \phi_{4}$ in terms of $\phi_{1}$ becomes impossible.

\subsection{An example of triangles with a side node}

To validate the results from equation (50) a sample triangle with a side node is analyzed. The (Mathematica) symbolic code (furnished in the Appendix) yielded the following shape functions.

$$
\begin{aligned}
\phi_{1} & =\frac{16}{9}-\frac{7 x}{18}-\frac{8 y}{9}+\frac{13 r}{18} \\
\phi_{2} & =-\frac{10}{63}+\frac{5 x}{63}+\frac{50 y}{63}-\frac{65 r}{63} \\
\phi_{3} & =-\frac{2}{7}+\frac{9 x}{14}-\frac{4 y}{7}+\frac{13 r}{42} \\
\phi_{4} & =-\frac{1}{3}-\frac{x}{3}+\frac{2 y}{3} \\
\text { where: } \quad r & =\sqrt{x^{2}-\frac{928 x}{169}+\frac{8 x y}{13}+\frac{16\left(\frac{1582}{1011}+y\right)\left(\frac{22219}{1312}+y\right)}{169}}
\end{aligned}
$$

The last three shape functions i.e., $\phi_{2}, \phi_{3}, \phi_{4}$ were computed analytically from the first one, $\phi_{1}$, using the interdependency relation stated in equation (50). It is intuitively obvious that the last shape function $\phi_{4}$, which contains only the linear terms but not the irrational term $r$, can never reproduce $\phi_{1}, \phi_{2}, \phi_{3}$ 
that contain $r$. The vanishing of the determinant associated with equation (50) furnishes an algebraic proof of this assertion.

\section{Benchmark examples}

To verify the results of computer programs to be developed in procedural programs the following cases - a general quadrilateral, trapezoid and parallelogram are executed by the symbolic computer program of Appendix-I.

\subsection{General quadrilateral}

The nodal coordinates are:

$$
\left(\frac{1}{5}, \frac{3}{10}\right) ;\left(\frac{11}{5}, \frac{3}{2}\right) ;\left(3, \frac{27}{10}\right) ;\left(\frac{7}{10}, 2\right)
$$

The isoparametric shape functions are:

$$
\begin{aligned}
\phi_{1} & =-\frac{9187}{5168}+\frac{2705 x}{2584}-\frac{3545 y}{2584}+\frac{55 r}{5168} \\
\phi_{2} & =-\frac{24521}{5168}-\frac{3555 x}{2584}+\frac{3035 y}{2584}-\frac{89 r}{5168} \\
\phi_{3} & =-\frac{9781}{2584}+\frac{2015 x}{1292}-\frac{1375 y}{1292}+\frac{35 r}{2584} \\
\phi_{4} & =\frac{2349}{1292}-\frac{795 x}{646}+\frac{815 y}{646}-\frac{1375 r}{1292} \\
r & =\sqrt{2500 x^{2}+3000 x y-66380 x+900 y^{2}+42860 y+78457}
\end{aligned}
$$

\subsection{Trapezoid}

The nodal coordinates for an example (like the trapezoid shown in Figure 6) are taken to be:

$$
\left(\frac{1}{5}, \frac{3}{10}\right) ;\left(\frac{11}{5}, \frac{3}{10}\right) ;\left(4, \frac{27}{10}\right) ;\left(\frac{710}{27} 10\right)
$$


The shape functions are:

$$
\begin{aligned}
\phi_{1} & =\frac{1}{4 \mathcal{D}}\left(-300 y^{2}+400 x y+20 y-1080 x+2133\right) \\
\phi_{2} & =\frac{1}{24 \mathcal{D}}\left(500 y^{2}-2400 x y-1020 y+6480 x-891\right) \\
\phi_{3} & =\frac{1}{24 \mathcal{D}}\left(-500 y^{2}+2400 x y-180 y-720 x+99\right) \\
\phi_{4} & =\frac{1}{4 \mathcal{D}}\left(300 y^{2}-400 x y+700 y+120 x-237\right) \\
\mathcal{D} & =441+130 y
\end{aligned}
$$

\subsection{Parallelogram}

The nodal points:

$$
\left(\frac{1}{10}, \frac{1}{10}\right) ;\left(\frac{13}{10}, \frac{2}{5}\right) ;\left(\frac{3}{2}, \frac{4}{5}\right) ;\left(\frac{3}{10}, \frac{1}{2}\right)
$$

for this example (of a typical parallelogram shown in Figure 7) led to the following computed shape functions:

$$
\begin{aligned}
\phi_{1} & =\frac{1}{147}\left(-100 x^{2}+450 y x-60 x-200 y^{2}-355 y+187\right) \\
\phi_{2} & =\frac{1}{294}\left(200 x^{2}-900 y x+330 x+400 y^{2}-130 y-17\right) \\
\phi_{3} & =\frac{1}{294}\left(-200 x^{2}+900 y x-50 x-400 y^{2}-10 y+3\right) \\
\phi_{4} & =\frac{1}{147}\left(100 x^{2}-450 y x-80 x+200 y^{2}+425 y-33\right)
\end{aligned}
$$

\section{Comparison with Wachspress shapes}

The brilliant idea of isoparametric shape functions of Taig was intuitive and lagged a rigorous geometrical basis. On the other hand Wachspress, vide ref. [12], developed shape functions for a class of finite elements based on 
the projective geometry constructs. A practical numerical scheme was published in ref. [5]. In the interest of complete treatment of the isoparametric formulation, an example of of a (convex) quadrilateral (four-node) element is furnished here. The difference between the isoparametric and the Wachspress formulations are studied using the close-form algebraic expressions for the shape functions.

It has been pointed out in ref. [5] that the isoparametric scheme exactly captures Wachspress' results for trapezoids (hence for parallelograms). Thus for the comparison a generic (irregular convex) quadrilateral is considered.

The quadrilateral element of Figure 2, with coordinates in (16), is considered. The Wachspress shape functions from ref. [5] (having $10^{-8}$ round off error with Mathematica Rationalize[results, 10-8] ) are:

$$
\begin{aligned}
& \psi_{1}=\frac{1}{\mathcal{D}}\left(\frac{116859}{10636}-\frac{5762 x^{2}}{3695}-\frac{5015 y}{112559}-\frac{34377 y^{2}}{10064}+-\frac{45835 x}{6292}+\frac{63082 x y}{10235}\right) \\
& \psi_{2}=\frac{1}{\mathcal{D}}\left(-\frac{6373}{8548}+\frac{11425 x^{2}}{10056}+x\left(\frac{60772}{9287}-\frac{31057 y}{7636}\right)-\frac{9576 y}{6199}+\frac{2836 y^{2}}{2583}\right) \\
& \psi_{3}=\frac{1}{\mathcal{D}}\left(\frac{998}{10003}-\frac{32437 x^{2}}{10901}-\frac{1657 y}{5680}-\frac{62349 y^{2}}{42745}-\frac{4971 x}{8875}+\frac{285850 x y}{48993}\right) \\
& \psi_{4}=\frac{1}{\mathcal{D}}\left(-\frac{48847}{39921}+\frac{24567 x^{2}}{7228}-x\left(\frac{28941}{9461}+\frac{57323 y}{7228}\right)+\frac{54407 y}{8893}+\frac{34287 y^{2}}{9079}\right) \\
& \mathcal{D}=\frac{94861}{10404}-\frac{59858 x}{13729}+\frac{20477 y}{4833}
\end{aligned}
$$

The difference $\chi=\psi-\phi$, from equations(54) and (59) are Taylor ex- 
panded up to quadratic terms, and the results are:

$$
\begin{aligned}
& \chi_{1}=\frac{2}{1151}-\frac{7 x}{823}-\frac{175 x^{2}}{3002}-\frac{5 y}{697}+\frac{83 x y}{769}+\frac{866 x^{2} y}{6063}-\frac{13 y^{2}}{720}-\frac{71 x y^{2}}{504}-\frac{45 x^{2} y^{2}}{296} \\
& \chi_{2}=-\frac{3}{1067}+\frac{17 x}{1235}+\frac{203 x^{2}}{2152}+\frac{7 y}{603}-\frac{164 x y}{939}-\frac{49 x^{2} y}{212}+\frac{22 y^{2}}{753}+\frac{106 x y^{2}}{465}+\frac{416 x^{2} y^{2}}{1691} \\
& \chi_{3}=\frac{3}{1357}-\frac{8 x}{739}-\frac{23 x^{2}}{310}-\frac{13 y}{1424}+\frac{118 x y}{859}+\frac{557 x^{2} y}{3064}-\frac{25 y^{2}}{1088}-\frac{258 x y^{2}}{1439}-\frac{107 x^{2} y^{2}}{553} \\
& \chi_{4}=-\frac{1}{879}+\frac{5 x}{898}+\frac{24 x^{2}}{629}+\frac{y}{213}-\frac{58 x y}{821}-\frac{79 x^{2} y}{845}+\frac{8 y^{2}}{677}+\frac{71 x y^{2}}{770}+\frac{81 x^{2} y^{2}}{814}
\end{aligned}
$$

These differences $\chi$ are of interest in problems where the secondary effects are of concern. High accuracy finite elements, as needed e.g., in stochastic finite element formulations and wave propagation problems, will yield significantly different results due to the differences denoted by $\chi$.

The four constant terms in equation (60) indeed dominate the difference $\chi$. A plotting exercise reveales that the first and third Wachspress shape functions are greater than those from the isoparametric case, whereas the corresponding second and the fourth ones yield relatively lower values.
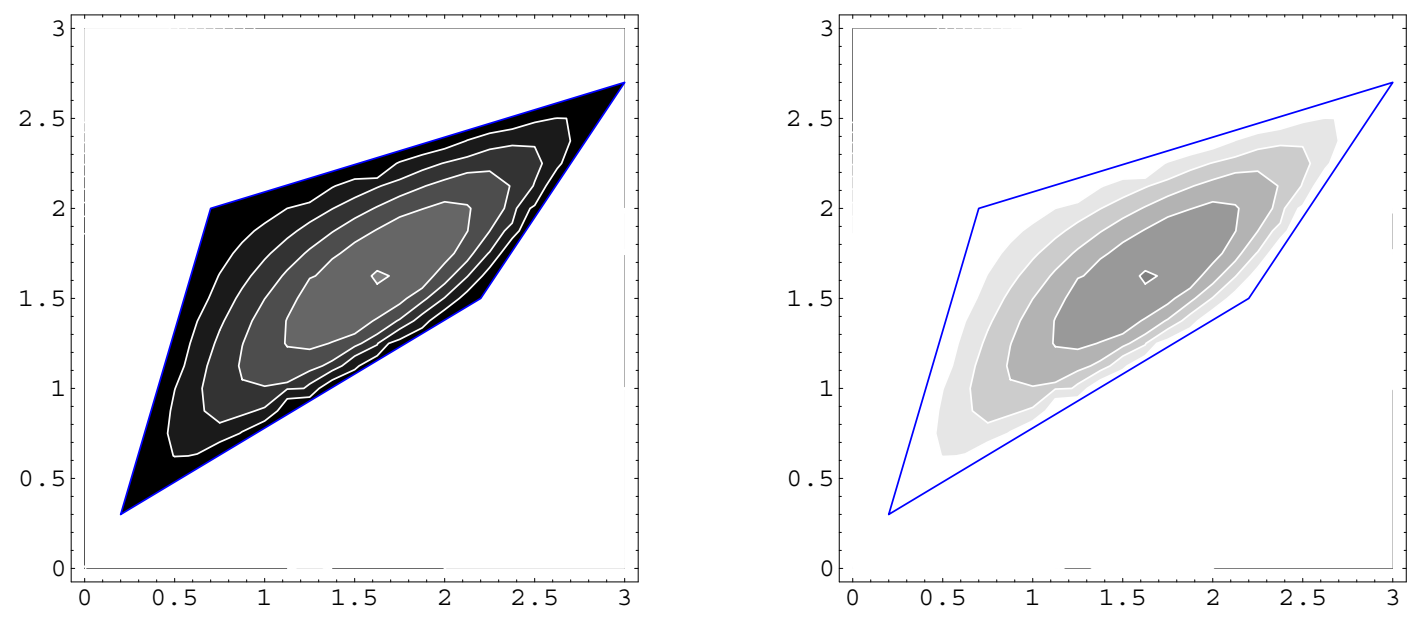

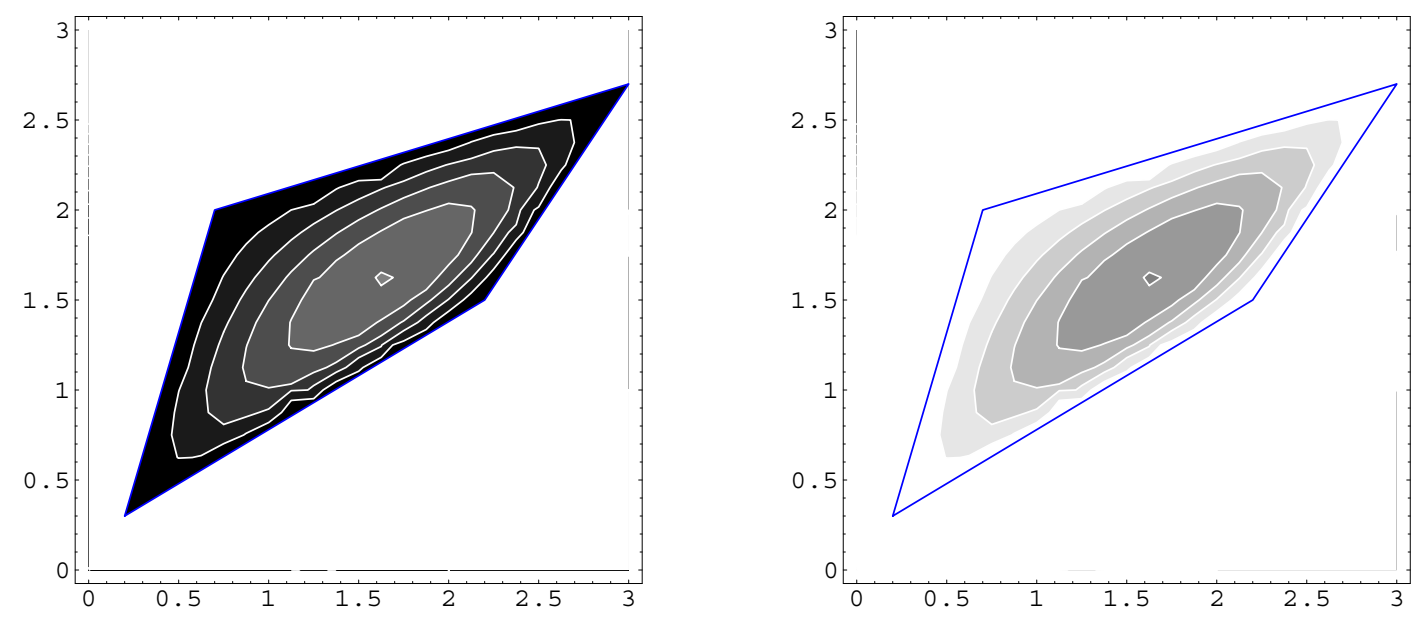

Figure 9: $\chi$-for the four shape functions; positive values in black background and negative values in light background

Since both isoparametric and the Wachspress shape functions are linear on the boundaries the difference is due to the inclusion of an arbitrary bubble function, vide ref. [13], due to the numerical artifact.

\section{FORTRAN or C programming}

Historically the FORTRAN language and $\mathrm{C}$ in the recent years have played the key role in succeeding and versatile implementation of a wide variety of finite element technics. Most commercial codes will necessitate modules that can be compiled and executed in a highly efficient manner. The symbolic nature of computer algebraic constructs has significantly enhanced the finite element development in an object oriented environment (e.g., $\mathrm{C}^{++}$or Java) 
where large applications can be managed under robust software engineering guidelines.

In a procedural environment, conventionally, the shape function are evaluated at isolated points. Numerical values of the Jacobian of the coordinate transformation $\left(J=\frac{\partial(x, y)}{\partial(\eta, \xi)}\right)$ are calculated to integrate in the $x$ - and $y-$ system by performing quadrature in $\eta-$ and $\xi-$ with $\mathrm{d} x \mathrm{~d} y$ replaced by $|J| \mathrm{d} \eta \mathrm{d} \xi$. Such programming modules (i.e., SUBROUTINE, FUNCTION) can be clearly coded by invoking two basic units:

1. determination of area of a triangle from nodal coordinates, as described in equation $(27)$

2. determination of a root $\eta$ or $\xi$

(a) for a general quadrilateral element:

from quadratic equations:

$$
\begin{gathered}
\alpha_{\eta} \eta^{2}+\beta_{\eta} \eta+\gamma_{\eta}=0 \text { as } \eta=\left(-\beta_{\eta}-\sqrt{\beta_{\eta}^{2}-4 \alpha_{\eta} \gamma_{\eta}}\right) / 2 \alpha_{\eta} \\
\alpha_{\xi} \xi^{2}+\beta_{\xi} \xi+\gamma_{\xi}=0 \text { as } \xi=\left(-\beta_{\xi}+\sqrt{\beta_{\xi}^{2}-4 \alpha_{\xi} \gamma_{\xi}}\right) / 2 \alpha_{\xi}
\end{gathered}
$$

Note the branches of the roots are based on counterclockwise numbering of nodes and the counterclockwise sense of $\eta$ to $\xi$.

(b) for a trapezoidal element:

$$
\beta \zeta+\gamma=0 \text { as } \zeta=-\gamma / \beta \text {; where } \zeta \text { stands for } \eta \text { or } \xi
$$

3. generation of the coefficients: $\alpha, \beta, \gamma$ from linear combinations of results of item 1. 


\section{Conclusions}

Closed form algebraic expressions for isoparametric shape functions permitted a deeper understanding of this versatile and extremely popular method, vide ref. [9]. Existing literature, both text books and research publications, hardly address the scope and limitation on a rigorous basis ref. [10] since they are (almost invariably) confined to shape functions expressed in terms of canonical coordinates $\eta$ and $\xi$ on a unit square $\left(\Omega^{\circ}\right)$. Widely available computer algebra systems can be easily employed to yield shape functions on arbitrary (convex) quadrilaterals $(\Omega)$ in terms of the physical $x$ and $y$ coordinate variables.

Closed form expressions reveal the important fact that, for trapezoids (hence parallelograms), the isoparametric results indeed coincide with the Wachspress' formulation based on projective geometry. This observation provides a basis to ascertain the improvement in accuracy of isoparametric elements as a pair of opposite sides tend to become more parallel.

The general form of shape functions in $x$ and $y$ coordinates contain square root terms, whereas those in the $\eta$ and $\xi$ system are strictly bilinear. This observation can provide a means to connect the intuitive isoparametric scheme with the Wachspress irrational shape functions. Furthermore, these radical subexpressions indicate that there cannot be a clear designation about the algebraic degree of interpolants since a Taylor expansion will contain all higher power in $x$ and $y$ terms. However, combination of linear terms and square root of quadratics indicate a first order representation. 
A set of new results in this paper indicate a procedure to construct the shape functions in $x$ and $y$ variables by solving quadratic equations. Very efficient procedural (C) and object oriented $\left(\mathrm{C}^{++}\right)$codes can be developed to construct shape functions by employing these relations in terms of areas of various (sub-)triangles within the quadrilateral finite element $(\Omega)$.

An advantage of using computer algebra is that one can visualize the singularity that emerges as a convex quadrilateral degenerates into a triangle with a side node. The closed form isoparametric shape functions still remain valid. It is of interest to explore how a square root term helps construct a function that remains zero on a semi-infinite straight line at the same time it grows linearly on its complement.

Exact integrations in $x$ and $y$ variables were addressed (in general terms by the author) in ref. [3]. Specific cases of irrational (square root type) integrands akin to the isoparametric formulation are addressed in ref. [6]. This method of computing, in $x$ and $y$ variables, addresses a number of crucial questions raised in the literature regarding the accuracy and anticipated convergence criteria, vide ref. [7], of solutions obtained from isoparametric quadrilateral finite elements. 


\section{Appendix: a Mathematica code}

isoparametricShapesAnalytical: : usage="isoparametricShapes [nodes, $\{\mathrm{x}, \mathrm{y}\}$ ]

returns shape functions in $\{x, y\}$ cordinates for a quadrilateral defined

by nodes containing the list of $\{x i, y i\}$ nodal locations.

Example: nodes $=\{\{1 / 5,3 / 10\},\{11 / 5,3 / 2\},\{3,27 / 10\},\{7 / 10,2\}\} "$

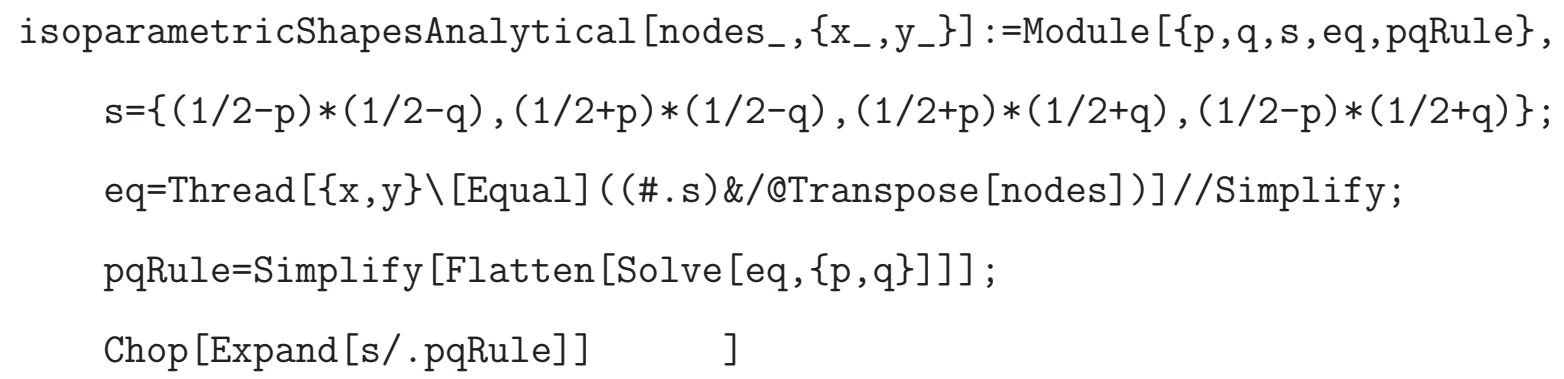




\section{Acknowledgement}

The research was supported by the following grants from the National Science Foundation: "Concave Finite Element Shape Functions" CMS-0202232, "Workshop for Scientists and Engineers on Structural Deformations at the Historic Site of Angkor, in Cambodia" OISE-0456406, and "US-France Cooperative Research: Engineering Shape Calculation for Surgery, Biology and Anthropology" INT-0233570.

\section{Dedication}

This paper is dedicated to the memory of the Late Professor Paresh N. Chatterjee, Emeritus Chair of the Applied Mechanics Department, Bengal Engineering College (Currently renamed as the Bengal Engineering and Science University), Shibpur, Howrah, West Bengal, India. He mentored two generations of researchers and educators of Engineering Mechanics and deeply touched the heart of every student and colleague in a humane way. 


\section{References}

[1] T. Belytschko and D. Lasry. A fractal patch test. International Journal for NumericalMethods in Engineering, 26(10):2199-2110, 1988.

[2] R. Courant. Variational methods for the solution of problems of equilibrium and vibration. Bulletin of the American Mathematical Society, 49:1-29, 1943.

[3] G. Dasgupta. Integration within polygonal finite elements. Journal of Aerospace Engineering, ASCE, 16(1):9-18, January 2003.

[4] G. Dasgupta. Interpolants within convex polygons: Wachspress' shape functions. Journal of Aerospace Engineering, ASCE, 16(1):1-8, January 2003.

[5] G. Dasgupta. Interpolants within convex polygons: Wachspress' shape functions. Journal of Aerospace Engineering, ASCE, 16(1):1-8, January 2003.

[6] G. Dasgupta. Stiffness matrix from isoparametric closed form shape functions using exact integration. Journal of Aerospace Engineering, ASCE, 2005. under review.

[7] Carlos A. Felippa, Bjørn Haugen, and Carmelo Militello. From the individual element test to finite element templates: Evolution of the patch test. International Journal for Numerical Methods in Engineering, 38:199-229, 1995. 
[8] D. F. Rogers and J. A. Adams. Mathematical Elements of Computer Graphics. McGraw-Hill, 1990.

[9] I. C. Taig. Structural analysis by the matrix displacement method. Report S017, English Electric Aviation Report, England, 1961.

[10] R.L. Taylor, J.C. Simo, O.C. Zienkiewicz, and A.C.H. Chen. The patch test - a condition for assessing FEM convergence. International Journal for Numerical Methods in Engineering, 22:39-62, 1986.

[11] E. L. Wachspress. A Rational Basis for Function Approximation, volume 228 of Lecture Notes in Mathematics. Springer Verlag, 1971.

[12] E. L. Wachspress. A rational finite element basis. Academic Press, 1975.

[13] O. C. Zienkiewicz and R. L. Taylor. The Finite Element Method, volume 2-Solid Mechanics. Elsevier, New York, NY, 5th edition, 2000. 